\title{
Quantitative ultrasound successes: past, present and future
}

\section{Michael Oelze}

Michael L. Oelze, "Quantitative ultrasound successes: past, present and future," Proc. SPIE 11319, Medical Imaging 2020: Ultrasonic Imaging and Tomography, 113190X (16 March 2020); doi: 10.1117/12.2557150 


\title{
Quantitative ultrasound successes: past, present and future
}

\author{
Michael L. Oelze ${ }^{\mathrm{a}}$ \\ ${ }^{a}$ Beckman Institute for Advanced Science and Technology, Department of Electrical and \\ Computer Engineering, University of Illinois at Urbana-Champaign, 405 N Mathews, Urbana, \\ IL, USA
}

\begin{abstract}
Diagnostic ultrasound is ubiquitous in clinical practice because it is safe, portable, inexpensive, has high spatial resolution and is real time. Therefore, improving the capabilities of diagnostic ultrasound is a highly significant clinically. In this talk we will discuss different applications of quantitative ultrasound (QUS) imaging and how QUS approaches have evolved over time. Specifically, we will discuss the use of spectral-based approaches to estimate the backscatter coefficient (BSC) and attenuation slope and the use of envelope statistics to describe underlying tissue microstructure. These QUS approaches have been successful at classifying tissue state, monitoring focused ultrasound therapy, detecting early response of breast cancer to neoadjuvant chemotherapy and the automatic detection of nerves in the imaging field. We will demonstrate how QUS approaches can be incorporated on breast tomography machines, which allow an expansion of the tradeoff between spatial resolution and the variance of QUS estimates. One of the ongoing issues with QUS is the inability to properly account for losses in tissues that affect the estimates of the backscatter coefficient. We will demonstrate new calibration procedures that can improve the ability to account for tissue losses. Finally, we will discuss how machine learning approaches can further improve QUS techniques by eliminating the need for models and in some cases eliminating the need for a reference scan.
\end{abstract}

Keywords: Ultrasound, Quantitative ultrasound, backscatter coefficient

\section{INTRODUCTION}

Ultrasound is ubiquitous in clinical medicine because it is inexpensive, portable, real time and considered the safe imaging modality. Ultrasound offers a variety of modes for both anatomical (e.g., B-mode) and functional (e.g., Doppler and color flow) imaging. More recent modes of imaging that have been added to clinical ultrasound devices are strain and shear elastography. These modes allow assessment of tissue mechanical properties with the goal of providing more quantitative information regarding tissue state.

Other quantitative ultrasound (QUS) modes have been under development for several years and have only recently been explored for adoption in ultrasound scanners for use in clinical medicine. Techniques that make use of the backscattered spectra through the backscatter coefficient (BSC) or techniques that quantify the statistics of the envelope have continued to make strides by demonstrating success in improving diagnostics. ${ }^{1,2}$ With improved technologies and demonstration of success of these techniques, the outlook for QUS techniques is very promising. Some roadblocks to implementation still exist, such as properly accounting for losses in the signals and the need for acquiring reference signals. However, these issues have been diminished over recent years with solutions to improve compensation of tissue losses and facilitate ease of implementation.

This paper briefly discusses the successes of QUS techniques both in the past, in the present and in the future. Foundational work in QUS will be highlighted followed by current research successes in QUS clinically. Finally, the future of QUS will be considered and how technical advances can broaden acceptance clinically.

Further author information: (Send correspondence to M.L.O)

M.L.O.: E-mail: oelze@illinois.edu, Telephone: 12173339226

Medical Imaging 2020: Ultrasonic Imaging and Tomography, edited by Brett C. Byram, Nicole V. Ruiter, Proc. of SPIE Vol. 11319, 113190X - () 2020 SPIE - CCC code: 1605-7422/20/\$21 - doi: 10.1117/12.2557150 


\section{PAST}

The BSC is defined as a backscattered cross section per unit volume from a scattering medium. ${ }^{3}$ The BSC is a fundamental tissue property and is a function of frequency. The BSC is usually represented is a spectrum and is calculated by taking the power spectrum of a scattered signal and dividing by a reference spectrum to account for system settings and field effects from the source. In this way, the BSC is considered an operator independent measure because over the same frequency band, the BSC from a medium can be replicated on different platforms assuming a correct accounting for the system effects through calibration. Several studies have demonstrated that the BSC can be consistently estimated across ultrasonic imaging platforms. ${ }^{4-7}$

QUS backscattering techniques based on the BSC have been successfully used to characterize different aspects of tissue microstructures. Noteworthy are the pioneering works of the mid-80s and early 90 s that demonstrated the ability of QUS based on spectral features to ultrasonically quantify ocular, liver, prostate, renal and cardiac tissues. ${ }^{8-10}$ The work of Lizzi et al. (Ref. 8) is especially notable because they developed the theoretical framework to implement a calibration spectrum approach to account for system-dependent effects.

If implemented correctly, spectral-based QUS using the BSC provide operator- and system-independent imaging biomarkers that can be used to diagnose disease or identify tissue state. In order to better quantify the BSC for diagnostic representation, the BSC is often parameterized using a model to describe the frequency dependence of the BSC. An approach used by Lizzi et al. was to parameterize the normalized backscatter power spectrum converted to a decibel scale by fitting a line to the normalized power spectrum versus frequency. ${ }^{11}$ The spectral slope, mid-band fit and spectral intercept were then used to parameterize the normalized power spectrum. Other approaches used models of tissue microstructure to derive features from the BSC related to the tissue microstructure. Specifically, based on the Born approximation for weak scattering, the BSC has been modeled using an intensity form factor approach. ${ }^{3,12,13}$ Based on the form factor models, estimates of scatterer properties (i.e., effective scatterer diameter (ESD) and effective acoustic concentration (EAC)) related to the underlying tissue microstructure are obtained. ${ }^{14}$

QUS imaging began to emerge, where color-coded images based on QUS parameter values were superimposed on B-mode images of different tissues structures. ${ }^{15,16}$ These images provided an immediate display of QUS parameters and how they could differentiate between different kinds of tissues. For example, Fig. 1 shows QUS images of three different kinds of rodent tumors. Using the appearance of the tumors in B-mode imaging alone did not provide sufficient diagnostic information to differentiate the benign tumors from the malignant tumors. However, the use of the ESD parameter provided clear separation between the benign tumors (Fig. 1, left) and the malignant cases (Fig. 1, middle and right). ${ }^{17}$

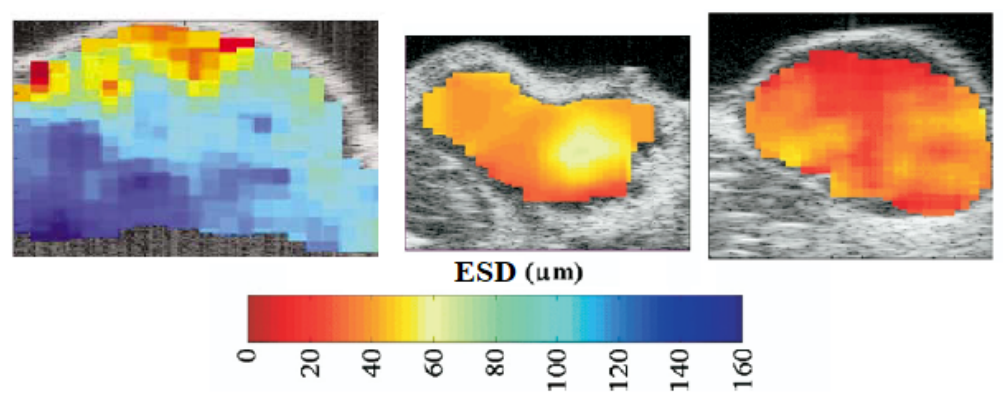

Figure 1. QUS images of three types of rodent tumors using the ESD parameter from a spherical Gaussian form factor. (Left) Benign fibroadenoma, (middle) malignant mammary carcinoma, (right) malignant mammary sarcoma.

\section{PRESENT}

In more recent work, i.e., in the last 10 years, several QUS successes have been able to move from laboratory animals to the clinic. A few of these successes are noted below. In most cases, QUS was first developed in an appropriate animal model for testing but was then translated to studies in humans with varying degrees of 
success. The translation of QUS from animals to humans was not without difficulty. However, the work over the past decade has paved the way for clinical implementation of QUS.

\subsection{Cancer Diagnostics and Therapy Monitoring}

QUS has been used to improve cancer diagnostics clinically. In one recent example, QUS was used predict the early response of breast cancer patients to neoadjuvant chemotherapy. ${ }^{18}$ Furthermore, in this study QUS parameters were used to predict survival of breast cancer patients. In the study, the ESD and EAC parameters were estimated from breast tumors at baseline, 1 week, 4 weeks, 8 weeks post initiation of chemotherapy and just before surgery. The EAC was observed to increase in patients responding to therapy as early as one week post treatment initiation, which further increased at week 4 and reached a maximum value at week 8 after therapy initiation. In contrast, non-responding patients did not exhibit changes in the QUS parameters. Figure 2 shows a graph of the EAC parameters versus days on the treatment regimen for both responders and nonresponders. The best predictor of treatment response was through the combination of EAC and ESD parameters at week 4 achieving a sensitivity of $82 \%$ and specificity of $100 \%$. The results demonstrate that spectral-based QUS parameters extracted from ultrasound data are predictive of ultimate clinical chemotherapy response, as early as 1 week post treatment initiation. The improved predictive capabilities provided by QUS could allow clinicians to further customize cancer therapies and to change ineffective treatments within days to weeks of starting therapy.

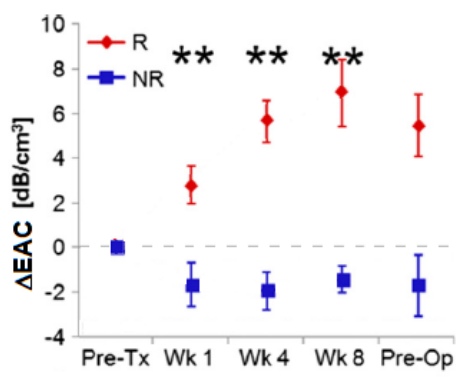

Figure 2. Graph of the average change in the EAC parameter per tumor estimated for breast cancer patients undergoing neoadjuvant chemotherapy for responders and nonresponders. ${ }^{* *}$ represents statisitcally signficant differences were observed between responders and nonresponders.

\subsection{Liver Disease}

Another application of QUS that has recently demonstrated success in in vivo animal studies and in clinical human studies is the ability of QUS to predict steatosis. ${ }^{19,20}$ In a study involving rabbits on a high fat diet, QUS techniques were able to classify between high and low lipid levels in the liver. ${ }^{20}$ Figure 3 shows plots of the ESD versus lipid level and the EAC versus lipid level. Similarly, in human clinical studies, QUS techniques were able to predict the grade of steatosis (grade 1, 2 or 3 ) with $68.3 \%$ accuracy. ${ }^{19}$
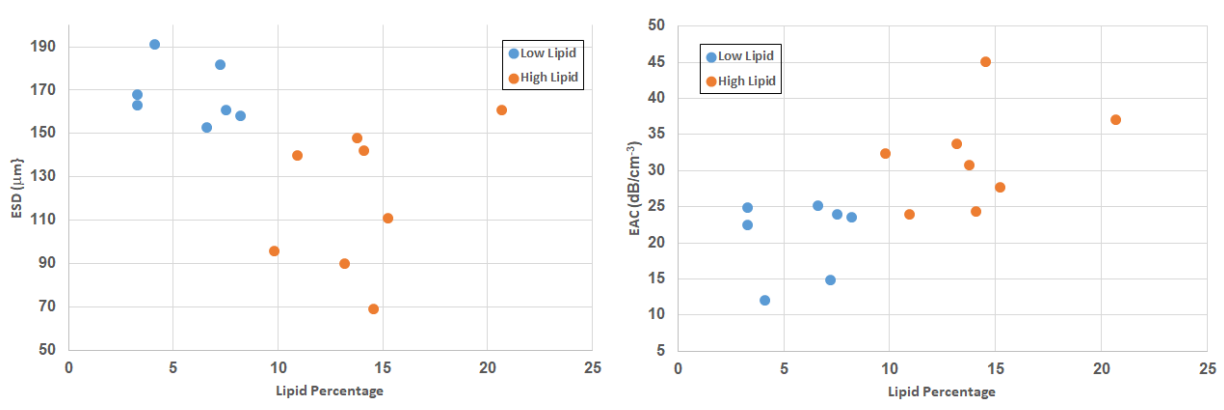

Figure 3. Graphs of the ESD versus lipid level and the EAC versus lipid level for rabbits on a high fat diet. 


\section{FUTURE}

Some of the remaining challenges associated with QUS are properly accounting for losses between the imaging probe and the region of interrogation, optimizing models to extract information from the signals and simplifying calibration procedures in the clinical workflow. The next three sections discuss possible approaches to remedying some of the remaining challenges.

\subsection{Model-Free and Reference-Free}

In a study on characterizing the liver of rabbits $(\mathrm{N}=57)$ on a high fat diet, ultrasonic scans of the rabbits were kept the same for each session. Therefore, we hypothesized that a 1D convolutional neural network (CNN) could separate the system-dependent signal from the tissue-dependent signal, and perform classification in a reference-free manner and without having to resort to a scattering model. A CNN learns a nonlinear mapping from the input to the output via stacking of multiple connected convolutional filter layers at different resolutions. The learned features from the convolutional layers can then be concatenated into a vector and classified by fully connected layers. In the case of ultrasonic tissue characterization, the problem was formulated as a supervised learning strategy using a CNN where the input was the backscattered radiofrequenyc (RF) data and the output was the pathological indicator (e.g. fatty/non-fatty).

The traditional spectral-based QUS approach does not utilize the phase information in the RF signal, because only the magnitudes of the power spectra from RF data were computed. We hypothesized that a CNN could extract classification power from the lost phase information from the time-domain RF data and perform feature extraction and classification simultaneously. In this study we compared the reference-free and model-free CNN approach with traditional QUS approaches that require a reference scan to classifying liver state and a model to extract parameters like the ESD and EAC. When classifying the liver state, i.e., below $5 \%$ lipid liver or above $5 \%$ lipid liver, classification accuracies were $59 \%$ when using QUS parameters with a support vector machine while the accuracy when using the CNN was $74 \%$. Therefore, the CNN provided better classification than traditional QUS methods that required both a model and reference spectra to be measured.

\subsection{In Situ Calibration}

One way to alleviate the need for a separate external calibration scan and to account for ultrasonic wave propagation losses incurred between the imaging array and the region of interrogation is to take advantage of a calibration target in the tissue. ${ }^{21}$ In the case of breast cancer imaging, before and during therapy, radiological clips are embedded in tumors and lymph nodes. Appropriately shaped clips could be used to provide a calibration spectrum for BSC estimation and to account for losses incurred. Figure 4 shows a B-mode image of a chicken breast sample having an overlying layer of fat on top and with a small 2-mm titatnium bead embedded in the tissue to act as a marker and as a calibration source. The BSC was calculated for the chicken breast using tradional reference phantom methods using external calibration targets and with the in situ calibration bead and compared. The results indicate the the BSC estimated using the in situ bead with a lossy layer on top was similar to the BSCs estimated without the lossy layer. The traditional reference phantom approach did not provide consistent BSC estimates.

\subsection{Combining with Ultrasound Computed Tomography}

Combining spectral-based QUS with ultrasound computed tomography (USCT) provides a means of accounting for system dependencies because the systems are already well calibrated. Furthermore, losses due to attenuation and transmission losses can be compensated because USCT provides maps of sound speed and attenuation. Finally, because the tomography devices rotate around the object, QUS estimates from multiple angles can be compounded reducing the variance of estimates and improving the trade off between estimate variance and spatial resolution. Figure 5 shows images from a tissue mimicking phantom scanned in a USCT system (Techniscan, Inc) with the sound speed image of the phantom, an uncorrected B-mode image of the phantom, a QUS ESD map of the phantom from a single view and finally a QUS ESD map made from compounding 64 angles. ${ }^{22}$ Combining QUS with USCT resulted in much better performance of QUS imaging with reduced variance of estimates and reduced bias because of attenuation correction. 

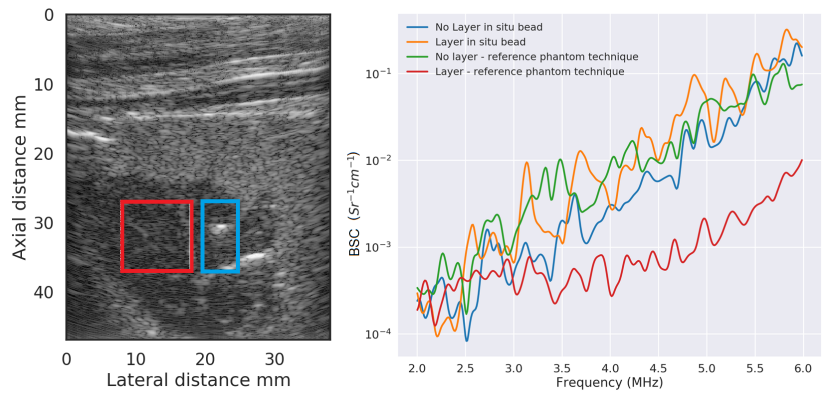

Figure 4. (Left) B-mode image of chicken breast with calibration bead and fatty layer on top and (right) BSCs estimated using traditional methods and in situ calibration target.
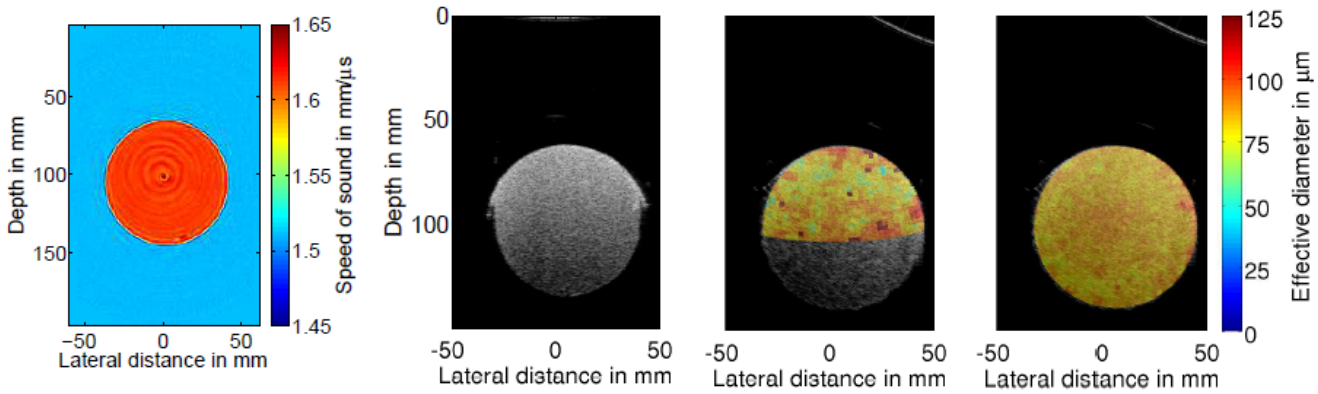

Figure 5. Tomography images of tissue mimicking phantom: (row 1) Sound speed image, (row 2) B-mode image with refraction artifact, (row 3) refraction corrected single shot QUS image and (row 4) compounded QUS image.

\section{CONCLUSION}

While QUS techniques based on the BSC and the spectrum of backscattered signals have been around for several decades, recent successes have allowed multiple clinical translations of these techniques. QUS has demonstrated the ability to detect response of breast cancer patients to chemotherapy, to quantify liver steatosis and to improve cancer diagnosis in the clinic. Future developments in QUS will be to eliminate the need for scattering models, reduce the need for external calibrations and improving performance by marrying the QUS technology with ultrasound computed tomography.

\section{ACKNOWLEDGMENTS}

The author would like to thank the following individuals that have helped in much of the work presented: William O'Brien, Jr., James Zachary, Rita Miller, Jim Blue, Roberto Lavarello, Jonatahn Mamou, Gooutam Ghoshal, Adam Luchies, Jeremy Kemmerer, Ernest Feleppa, Trong Nguyen and Anthony Podkowa. This work has been funded over the years by grants from the NIH (F32CA96419, R43CA121521, R01EB008992, R01CA111289, R21CA139095, R21EB020766).

\section{REFERENCES}

[1] Mamou, J. and Oelze, M. L., [Quantitative ultrasound in soft tissues], Springer (2013).

[2] Oelze, M. L. and Mamou, J., "Review of quantitative ultrasound: Envelope statistics and backscatter coefficient imaging and contributions to diagnostic ultrasound," IEEE transactions on ultrasonics, ferroelectrics, and frequency control 63(2), 336-351 (2016).

[3] Insana, M. F., Wagner, R. F., Brown, D. G., and Hall, T. J., "Describing small-scale structure in random media using pulse-echo ultrasound," The Journal of the Acoustical Society of America 87(1), 179-192 (1990).

[4] Wear, K. A., Stiles, T. A., Frank, G. R., Madsen, E. L., Cheng, F., Feleppa, E. J., Hall, C. S., Kim, B. S., Lee, P., O'Brien Jr, W. D., et al., "Interlaboratory comparison of ultrasonic backscatter coefficient measurements from 2 to $9 \mathrm{mhz}, "$ Journal of ultrasound in medicine 24(9), 1235-1250 (2005). 
[5] Wirtzfeld, L. A., Ghoshal, G., Hafez, Z. T., Nam, K., Labyed, Y., Anderson, J. J., Herd, M.-T., Haak, A., He, Z., Miller, R. J., et al., "Cross-imaging platform comparison of ultrasonic backscatter coefficient measurements of live rat tumors," Journal of Ultrasound in Medicine 29(7), 1117-1123 (2010).

[6] Nam, K., Rosado-Mendez, I. M., Wirtzfeld, L. A., Ghoshal, G., Pawlicki, A. D., Madsen, E. L., Lavarello, R. J., Oelze, M. L., Zagzebski, J. A., O'Brien Jr, W. D., et al., "Comparison of ultrasound attenuation and backscatter estimates in layered tissue-mimicking phantoms among three clinical scanners," Ultrasonic imaging 34(4), 209-221 (2012).

[7] Wirtzfeld, L. A., Nam, T. A., Labyed, Y., Ghoshal, G., Haak, A., Sen-Gupta, E., He, Z., Hirtz, N. R., Miller, R. J., Sarwate, S., et al., "Techniques and evaluation from a cross-platform imaging comparison of quantitative ultrasound parameters in an in vivo rodent fibroadenoma model," IEEE transactions on ultrasonics, ferroelectrics, and frequency control 60(7), 1386-1400 (2013).

[8] Lizzi, F. L., Greenebaum, M., Feleppa, E. J., Elbaum, M., and Coleman, D. J., "Theoretical framework for spectrum analysis in ultrasonic tissue characterization," The Journal of the Acoustical Society of America 73(4), 1366-1373 (1983).

[9] Madaras, E. I., Barzilai, B., Perez, J., Sobel, B., and Miller, J., "Changes in myocardial backscatter throughout the cardiac cycle," Ultrasonic imaging 5(3), 229-239 (1983).

[10] Insana, M. F., Hall, T. J., and Fishback, J. L., "Identifying acoustic scattering sources in normal renal parenchyma from the anisotropy in acoustic properties," Ultrasound in Medicine and Biology 17(6), 613626 (1991).

[11] Lizzi, F. L., Ostromogilsky, M., Feleppa, E. J., Rorke, M. C., and Yaremko, M. M., "Relationship of ultrasonic spectral parameters to features of tissue microstructure," IEEE transactions on ultrasonics, ferroelectrics, and frequency control 34(3), 319-329 (1987).

[12] Nicholas, D., "Evaluation of backscattering coefficients for excised human tissues: results, interpretation and associated measurements," Ultrasound in Medicine 83 Biology 8(1), 17-28 (1982).

[13] Nassiri, D. and Hill, C., "The use of angular acoustic scattering measurements to estimate structural parameters of human and animal tissues," The Journal of the Acoustical Society of America 79(6), 20482054 (1986).

[14] Oelze, M. L., Zachary, J. F., and O'Brien Jr, W. D., "Characterization of tissue microstructure using ultrasonic backscatter: Theory and technique for optimization using a gaussian form factor," The Journal of the Acoustical Society of America 112(3), 1202-1211 (2002).

[15] Oelze, M. L., Zachary, J. F., and O'Brien Jr, W. D., "Parametric imaging of rat mammary tumors in vivo for the purposes of tissue characterization," Journal of Ultrasound in Medicine 21(11), 1201-1210 (2002).

[16] Oelze, M. L., O'Brien, W. D., Blue, J. P., and Zachary, J. F., "Differentiation and characterization of rat mammary fibroadenomas and $4 \mathrm{t} 1$ mouse carcinomas using quantitative ultrasound imaging," IEEE Transactions on medical imaging 23(6), 764-771 (2004).

[17] Oelze, M. L., "Quantitative ultrasound techniques and improvements to diagnostic ultrasonic imaging," in [2012 IEEE International Ultrasonics Symposium], 232-239, IEEE (2012).

[18] Sannachi, L., Tadayyon, H., Sadeghi-Naini, A., Tran, W., Gandhi, S., Wright, F., Oelze, M., and Czarnota, G., "Non-invasive evaluation of breast cancer response to chemotherapy using quantitative ultrasonic backscatter parameters," Medical image analysis 20(1), 224-236 (2015).

[19] Paige, J. S., Bernstein, G. S., Heba, E., Costa, E. A., Fereirra, M., Wolfson, T., Gamst, A. C., Valasek, M. A., Lin, G. Y., Han, A., et al., "A pilot comparative study of quantitative ultrasound, conventional ultrasound, and mri for predicting histology-determined steatosis grade in adult nonalcoholic fatty liver disease," American Journal of Roentgenology 208(5), W168-W177 (2017).

[20] Nguyen, T. N., Podkowa, A. S., Tam, A. Y., Arnold, E. C., Miller, R. J., Park, T. H., Do, M. N., and Oelze, M. L., "Characterizing fatty liver in vivo in rabbits, using quantitative ultrasound," Ultrasound in medicine Ej biology (2019).

[21] Nguyen, T. N., Tam, A. J., Do, M. N., and Oelze, M. L., "Estimation of backscatter coefficients using an in situ calibration source," IEEE Transactions on Ultrasonics, Ferroelectrics, and Frequency Control (2019).

[22] Lavarello, R. J., Oelze, M. L., Berggren, M., Johnson, S., Orescanin, M., and Yapp, R., "Implementation of scatterer size imaging on an ultrasonic breast tomography scanner," in [2009 IEEE International Ultrasonics Symposium], 305-308, IEEE (2009). 